\title{
La evaluación formativa en álgebra a través de los medios formales, semiformales $y$ no formales: el caso de expresiones $y$ polinomios algebraicos
}

Daniel Muñoz Suárez ${ }^{\mathrm{a}}$ ， María Vanessa Galindo Ramírez ${ }^{\mathrm{b}}$ ，Yair Martinez Martinez ${ }^{a}$ Facultad de educación, Universidad de Antioquia. daniel.msuarez@gmail.com ${ }^{b} F a c u l t a d ~ d e$ educación, Universidad de Antioquia. mvanessa.galindo@udea.edu.co ${ }^{\mathrm{c}} \mathrm{Facultad}$ de educación, Universidad de Antioquia. yair.martinez@udea.edu.co

\begin{abstract}
Resumen
El presente trabajo describe la relación entre la satisfacción percibida por los estudiantes con los medios de evaluación, en función de los logros y el rendimiento académico al adquirir competencias y formación en expresiones y polinomios algebraicos. Es por lo anterior, que la evaluaciòn se convierte en el eje fundamental de la estrategìa metodologica para la enseñanzaaprendizaje, requiriendo señalar que ambas no tienen objetivos separados ni ausentes una de la otra; es decir, que no se entenderán como la consecución de un procedimiento que está constituido por la planeación, la ejecución y finalmente por su posterior "evaluación"; sino en su lugar se considera un proceso de evaluación permanente donde ésta se transversaliza con la enseñanza y el aprendizaje, modificando la didáctica, la pedagogía y puntualizando la concepción misma de evaluación. Anotando entonces, que la evaluación cuando se refiere a la educación, no se debe de mirar únicamente con calificativos cuantitativos o cualitativos, sino que debe partir de un carácter formativo y reflexivo, el cual permite reconocer el conocimiento adquirido. En concreto, la evaluación puede valerse de instrumentos que arrojen resultados numéricos, pero teniendo en cuenta los medios y técnicas propios de ella, para propiciar el alcance de un aprendizaje significativo.
\end{abstract}

Palabras clave: Educación, evaluación, medios de evaluación, aprendizaje

\footnotetext{
Abstract

The main objective is this research is to describe the existing relationship between the students' satisfaction and assessment strategies towards to learning goals and academic performance, when acquiring competences and learning about algebraic expressions and polynomials. Considering these values to be central axes for assessment and their role in the methodology of teaching-learning. In that case, it does not have to be separated or absent objectives, as commonly understood that specific methodology, which means that, it is a step towards achieving, made by planning, implementing, and finally, evaluating. This is also understood as a process of continuous assessment where it runs across the teaching and learning process, meanwhile, modifying the didactics, the pedagogy and highlighting the very conception of evaluation. Thus, assessment in the educational field does not
} 
La evaluación formativa en álgebra a través de los medios formales, semiformales y no formales: el caso de expresiones y polinomios algebraicos

have to be seen only as qualitative and quantitative methods, but, it has to start from formative and reflective techniques that enables us to recognise the acquired knowledge. Consequently, assessment can be done from instruments that show numerical results, but, taking into account specific strategies and techniques to promote the scope of meaningful learning.

\section{Keywords: Education, assessment, assessment means, teaching, learning,} algebraic polynomials

\section{Introducción}

La evaluación en el ámbito educativo es uno de los temas que ha tomado fuerza en la actualidad y no porque sea una problemática nueva, sino porque ésta evidencia el aprendizaje logrado por el estudiante. Se debe señalar entonces, que en los procesos evaluativos se han generado innumerables definiciones y concepciones de la misma, lo cual refleja confusión con respecto a ella y sus diferentes medios y elementos, del tal manera que su concepción suele ser reconocida y tiende a reducirse al examen con su calificación, ya sea de carácter cualitativo o cuantitativo, donde los resultados suelen constituirse en una lógica boleana de cumple o no cumple con el procedimiento, sin atender a las competencias que involucra y a los logros implicados en el desarrollo de los estudiantes.

Puntualizando en lo anterior, se debe tener en cuenta que evaluar va más allá de un examen con lápiz y papel, implica una actividad continua y formativa, de tal modo que le permite al maestro detectar las dificultades que pueden estar presentes en el estudiante al momento de establecer si éste ha alcanzado los logros de aprendizaje esperados. Al respecto conviene decir que la evaluación se propone detectar cuales falencias se encuentran en los educandos, para poder hacer las correcciones pertinentes durante la enseñanza, de tal manera que ésta sea formativa y permita constituir y consolidar el aprendizaje a través de estrategias de mejora. Es así, como el estudiante reconoce la importancia de la evaluación para su formación, entendiendo que no es un proceso externo a él, sino que requiere de su participación y disposición para alcanzar los logros propuestos. Así pues, la evaluación no puede ir en una sola dirección, debe de variar según como resulte más beneficiosa para el individuo, por tanto es indispensable conocer y saber aplicar un sistema de evaluación continuo, formativo y diverso en metodologías de enseñanza-aprendizaje, que posibiliten múltiples alternativas de trabajo mediante estrategias y oportunidades que le permitan al estudiante adquirir competencias en el hacer, saber hacer y el ser.

En diferentes estudios que involucran prácticas de evaluación, se consideran diferentes perspectivas para definir las valoraciones de la evaluación de los aprendizajes. De esta variedad de posibilidades, el desarrollo de la práctica docente implica tomar en consideración la incorporación de nuevas prácticas de enseñanza y nuevos modelos de evaluación, para que en dicho proceso se demanden habilidades de comprensión, aptitud para las labores prácticas y la creatividad de los estudiantes, en lugar de mecanismos operativos supeditados a la memoria (Colclough, 2005).

El docente entonces, planifica en función de la evaluación del proceso y de las necesidades 
que éste involucre, considerando como activo el papel de sus participantes a través de diversas estrategias que apuntan a la consolidación del aprendizaje. Así pues, la planificación y el desarrollo de la práctica docente en este caso surge desde la determinación de los medios de evaluación, y por tanto, de todo lo que estos implican. Rodríguez e Ibarra (2012) afirman: "Entendemos por medio de evaluación cualquier prueba o evidencia que sirve para recabar información sobre el objeto a evaluar" (p. 9).

Los medios evaluativos pueden ser confundidos con las técnicas evaluativas o con los instrumentos evaluativos, los mismos medios podrían pensarse desde la frecuencia del uso del medio en el aula de clase, o desde lo convencional que pueda resultar dicho medio en el aula de clase o para una asignatura en particular, dado que en matemáticas no es usual usar medios evaluativos comunes para las ciencias sociales. En la literatura los medios evaluativos pueden codificarse como formales, semiformales y no formales desde en nivel de planeación que involucra; a saber pues, se concibe desde la planeación los medios formales, estos "exigen un proceso de planeación y elaboración más sofisticado y suelen aplicarse en situaciones que demanden un mayor grado de control" (Genovard y Gotzens, 1990, citado por Díaz-Barriga y Hernández, 2002), como ejemplo pueden ser: los exámenes, tests diagnóstico,ensayos, entre otros; los medios semiformales además de necesitar un periodo de preparación, precisan tiempo para su valoración y exhorta a los estudiantes respuestas más duraderas, lo que implica asignar una calificación (Díaz y Barriga, 2002, p.372), como ejemplo pueden ser: las prácticas supervisadas, exposiciones, debates, etcétera; los medios informales "se utilizan dentro de episodios de enseñanza con una duración breve, (...) exigen poco gasto didáctico, pueden utilizarse a discreción en la misma situación de enseñanza y aprendizaje" (Díaz y Barriga, 2002, p.367), como ejemplo pueden ser: los cuestionamientos orales y salir al tablero a resolver un ejercicio que se derive de una pregunta de clase.

En consecución, para evaluar el proceso de enseñanza-aprendizaje desarrollado por los estudiantes individualmente y en conjunto, partiendo desde los medios evaluativos mencionados, se considera necesariamente: el objeto de la evaluación, las técnicas de evaluación, los instrumentos de evaluación, las modalidades de evaluación, e incluso el método de enseñanza-aprendizaje y las competencias que implican.

La elección del medio evaluativo y del método de enseñanza aprendizaje, surge desde la determinación del objeto de la evaluación, entendiendo por objeto de evaluación aquello que se evalúa. La definición de objeto a evaluar en el campo educativo, afecta la selección de los métodos sobre los procesos de enseñanza y aprendizaje, dado que el objeto de evaluación busca definir las estrategias y los procedimientos que permitirán a los alumnos lograr los objetivos planteados por el profesor y el currículum, según los conocimientos, habilidades y actitudes incluidos en los objetivos del aprendizaje; por lo tanto, se parte desde la planeación de la enseñanza, dado que algunos métodos son más apropiados para evaluar el dominio cognitivo-de desempeño y otros el afectivo-de disposición, consolidando una estrecha relación entre la enseñanza y la evaluación, facilitando de este modo la retroalimentación y la comunicación sobre dichos procesos de enseñanza y aprendizaje (García, Aguilera, Pérez y Muñoz, 2011). 
La evaluación formativa en álgebra a través de los medios formales, semiformales y no formales: el caso de expresiones y polinomios algebraicos

Las técnicas son: " estrategias que el profesorado utiliza para recoger información acerca de las producciones y evidencias creadas por el alumnado (de los medios)"(Hamodi, y otros. 2015. p.158); "estrategias que utiliza el evaluador para recoger sistemáticamente información sobre el objeto evaluado. Pueden ser de tres tipos, la observación, la encuestación (entrevistas) y el análisis documental y de producciones" (Rodríguez e Ibarra, 2011, p.71-72); autoinformes, observación de otros colegas, observación en el aula, análisis de documentos y producciones, cuestionarios de evaluación, entrevistas (individual/grupal) y análisis del aprendizaje (Rodríguez e Ibarra, 2012).

Los instrumentos hará referencia a: "las herramientas que tanto los profesores como los alumnos utilizan para plasmar de manera organizada la información recogida mediante una determinada técnica de evaluación" (Hamodi, y otros. 2015. p.158); "herramientas reales y tangibles utilizadas por la persona que evalúa para sistematizar sus valoraciones sobre los diferentes aspectos" (Rodríguez e Ibarra, 2011, p.71-72). En definitiva, a través de un instrumento de evaluación, el evaluador concreta su valoración sobre el objeto evaluado, especificando la presencia o ausencia de una determinada característica, el grado en que ésta se da o las condiciones y características que la determinan. Como ejemplos de instrumentos de evaluación podemos citar las escalas de valoración, listas de control, diferenciales semánticos, cuestionarios o rúbricas (Rodríguez e Ibarra, 2012, p-11).

Las modalidades de evaluación van más allá de cómo evalúa el profesor el proceso del estudiante tanto de manera cualitativa como cuantitativa, de hecho este tipo de modalidad se conoce como heteroevaluación; sin embargo esta modalidad tiene otras posibilidades, como cuando es realizada por compañeros, conociéndose como coevaluación o evaluación entre pares (o iguales). Ibarra y Rodríguez (2014) afirman: Las modalidades participativas de evaluación implican considerar la dicotomía entre heteroevaluación (evaluación a otro o diferente) y autoevaluación (evaluación de sí mismo), y el grado de participación del estudiante en la valoración del trabajo, producto o actuación propio o de los compañeros en este proceso (autoevaluación, evaluación entre iguales o la coevaluación). "La autoevaluación implica un proceso mediante el cual los aprendices realizan un análisis y valoración de sus actuaciones y/o sus producciones" (Ibarra y Rodríguez, 2014, p.343); "la evaluación entre iguales implica un proceso mediante el cual los aprendices realizan un análisis y valoración sobre las actuaciones o producciones de aprendizaje desarrollados por parte de todos, de algún grupo o bien de algún estudiante" (Rodríguez e Ibarra, 2011, citado por Ibarra y Rodríguez, 2014, p.343); la coevaluación implica un proceso mediante el cual los docentes, junto con los aprendices, realizan un análisis y valoración de forma colaborativa,conjunta y consensuada sobre las actuaciones, producciones y/o productos de aprendizaje (Ibarra y Rodríguez, 2014, p.344).

Como la evaluación se da durante todo el proceso de enseñanza, el alcance del aprendizaje se constituye como una variable dependiente de la enseñanza y de la evaluación misma, lo que conlleva a que es posible seleccionar uno o varios medios evaluativos y por tanto algún método de enseñanza-aprendizaje correspondiente en un momento determinado del proceso según la planificación realizada y ejecutada; se tienen opciones de aprendizaje basadas en: experiencias, indagación, juegos, situación problema, proyectos, compartido y cooperativo, 
expositivo, investigación-acción, globalizado, por descubrimiento, modelamiento, por estudio de casos, VAK (visual, auditivo y kinestésico), entre otras. Desde este ángulo, existen medios evaluativos formales, semiformales e informales que pueden considerarse para determinar el método de enseñanza-aprendizaje a desarrollar con los estudiantes, fundamentando el mejoramiento a través del trabajo y el fortalecimiento de sus capacidades.

Las competencias en matemáticas según el Ministerio de Educación Nacional (1998) de Colombia son tres: Conocimientos básicos (pensamiento numérico y sistemas numéricos, pensamiento variacional y sistemas algebraicos y analiticos, pensamiento métrico y sistemas de medida, pensamiento espacial y sistemas geométricos, y pensamiento aleatorio y sistema de datos), procesos (razonamiento, resolución y planteamiento de problemas, comunicación, modelación, y elaboración-comparación y ejercitación de procedimientos) y contexto (situaciones problemáticas, de las matemáticas, de la vida diaria, de las otras ciencias).

En relación con todo lo anterior, el tema disciplinar a desarrollar en el aula fue el álgebra escolar, la cual se concibe como una rama de las matemáticas de carácter indispensable, necesario y obligatorio a impartir en las instituciones educativas, tanto por su utilidad en la vida diaria como por las capacidades, habilidades, competencias y tipos de pensamientos que se espera desarrollen los educandos. "La transición de la aritmética al álgebra es un paso importante para llegar a ideas más complejas y abstractas dentro de las matemáticas escolares" (Pérez, Pérez, y Hernández, 2013, p.863).

El eje central de enseñanza en nuestro caso es el álgebra, particularmente las expresiones y polinomios algebraicos. El diseño y adecuación de cada medio evaluativo a esta temática y sus objetos de evaluación, corresponden a parte de la investigación que venimos desarrollando para aspirar al título de licenciados en matemáticas y física. El trabajo de grado está realizándose entre el año 2018 y 2019 en la Universidad de Antioquia, Colombia.

Dado todo este panorama, la pregunta de investigación sustenta este artículo es: ¿en el caso de estudio de expresiones y polinomios algebraicos cómo se relaciona la satisfacción por la evaluación formativa con los logros alcanzados por los estudiantes al aplicarles medios formales, no formales y semiformales en el proceso de enseñanza-aprendizaje?

\section{Objetivos}

Este trabajo se propone correlacionar el grado de satisfacción que tienen los estudiantes por los medios evaluativos (formales, informales y no formales) con el grado de mejoramiento académico que estos alcanzan cuando se estudia las expresiones y los polinomios algebraicos; así como también analizar y determinar los logros de aprendizaje alcanzados por los estudiantes en relación a las competencias de formación cuando se aplica la evaluación formativa a partir de los medios evaluativos seleccionados y sus correspondientes técnicas, instrumentos y modalidades.

Las hipótesis que se plantean por tanto para verificar los objetivos de esta investigación

(cc) EY-NC-ND 2019, Universitat Politècnica de València 
son: hipótesis 1: A mayor satisfacción de los estudiantes por la evaluación formativa al emplear medios evaluativos formales, semiformales y no formales se genera aumento en los logros alcanzados y en el rendimiento académico; hipótesis 2: Cuando se emplean únicamente medios tradicionales formales de evaluación el rendimiento académico no mejora de igual manera y el grado de satisfacción es menor.

\section{Desarrollo de la innovación}

Al asumir la tarea de planificar la enseñanza del tema de estudio correspondiente (expresiones y polinomios algebraicos) desde la evaluación formativa, se pretendió abarcar diferentes necesidades y estímulos formativos para obtener logros de aprendizaje y mejoras para los estudiantes, a partir de medios que retroalimentaran, proalimentaran (que reaccionan a los cambios del contexto del proceso educativo) y que reflexionan el objeto de estudio, para buscar mejorar los resultado obtenidos y deseados. Se estableció una secuencia de medios evaluativos (formales, semiformales y no formales) en el proceso de enseñanza para los fines propuestos, por lo que hubo que pensar también: el objeto de la evaluación, la técnica que le corresponde, así como el instrumento, la modalidad y el método de enseñanza-aprendizaje para cada caso considerado.

La siguiente matriz se diseñó para realizar la práctica anteriormente mencionada, combinando información entre columnas y filas para describir la planeación de la enseñanza a través de la evaluación; se caracterizó por colores correspondientes a medios evaluativos, técnicas, instrumentos y modalidades con respecto a la evaluación. Los componente del objeto de evaluación no se describen dado que requieren de una extensión más extensa del escrito, habría por tanto que inferir que corresponde a cada objeto según lo anteriormente expuesto.

\begin{tabular}{|c|c|c|c|c|c|}
\hline \multirow{2}{*}{ SECUENCIA } & \multirow{2}{*}{ ОВJETO } & \multicolumn{3}{|c|}{ MEDIO } & TÉCNICA \\
\hline & & FORMAL & \begin{tabular}{|c} 
SEMIFORMA \\
L
\end{tabular} & \begin{tabular}{|c}
$\begin{array}{c}\text { INFORMA } \\
\mathbf{L}\end{array}$ \\
\end{tabular} & \\
\hline \multirow{3}{*}{1} & \multirow{9}{*}{$\begin{array}{l}\text { Tema: }\{A, B, C, \ldots\} \\
\text { I. Criterios: } \\
\text { a. Conocimientos } \\
\text { b. Habilidades } \\
\text { c. Actitudes } \\
\text { II. Competencias } \\
\text { III. Propósito u } \\
\text { objetivo de } \\
\text { aprendizaje } \\
\text { (logro) }\end{array}$} & & \multirow{3}{*}{$\begin{aligned} & \text { Medio 1 } \\
& \rightarrow \text { Método de } \\
& \text { enseñanza- } \\
& \text { aprendizaje 1 }\end{aligned}$} & \multirow{3}{*}{\begin{tabular}{|l|}
\multicolumn{1}{|c|}{ Medio 3 } \\
$\rightarrow$ Método de \\
enseñanza- \\
aprendizaje 3 \\
\end{tabular}} & Técnica 1 \\
\hline & & & & & Instrumento 1 \\
\hline & & & & & Modalidad 1 \\
\hline 2 & & \multirow{3}{*}{$\begin{aligned} & \text { Medio 2 } \\
& \rightarrow \text { Método de } \\
& \text { enseñanza- } \\
& \text { aprendizaje } 2 \\
&\end{aligned}$} & & \multirow{3}{*}{ 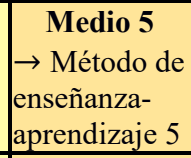 } & Técnica 2 \\
\hline- & & & & & Instrumento 2 \\
\hline 3 & & & & & Modalidad 2 \\
\hline \multirow[t]{3}{*}{-} & & & \multirow{3}{*}{\begin{tabular}{|l}
$\quad$ Medio 4 \\
$\rightarrow$ Método de \\
enseñanza- \\
aprendizaje 4
\end{tabular}} & & Técnica 3 \\
\hline & & & & & Instrumento 3 \\
\hline & & & & & Modalidad 3 \\
\hline
\end{tabular}

Tabla 1. Matriz modelo con ejemplos para el diseño de la evaluación 


\begin{tabular}{|c|c|c|c|c|c|}
\hline \multirow{3}{*}{$\begin{array}{l}\mathrm{S} \\
\mathrm{e} \\
\mathrm{c} \\
\mathrm{u} \\
\mathrm{e} \\
\mathrm{n} \\
\mathrm{c} \\
\mathrm{i} \\
\mathrm{a}\end{array}$} & \multirow{3}{*}{ ОВJETO } & \multirow{2}{*}{\multicolumn{3}{|c|}{ MEDIO }} & TÉCNICA \\
\hline & & & & & INSTRUMENTO \\
\hline & & FORMAL & SEMIFORMAL & INFORMAL & MODALIDAD \\
\hline \multirow{6}{*}{1} & \multirow{6}{*}{$\begin{array}{l}\text { Tema: } \\
\text { Necesidad e } \\
\text { importancia del } \\
\text { Álgebra }\end{array}$} & & & \multirow{3}{*}{$\begin{array}{l}\text { 1. Preguntas de } \\
\text { clase - 2. Discusión } \\
\text { grupal } \\
\text { Aprendizaje por } \\
\begin{array}{lr}\text { descubrimiento } \\
\text { indagación }\end{array}\end{array}$} & Observación \\
\hline & & & & & Rúbrica \\
\hline & & & & & Heteroevaluación \\
\hline & & \multirow{3}{*}{\begin{tabular}{|l} 
3. Plickers \\
Aprendizaje \\
basado por \\
juegos
\end{tabular}} & \multirow{3}{*}{$\begin{array}{l}\text { 4. Diapositivas } \\
\text { Aprendizaje } \\
\text { guiado y basado } \\
\text { por juego }\end{array}$} & & Encuestación \\
\hline & & & & & Cuestionario \\
\hline & & & & & Heteroevaluación \\
\hline \multirow[t]{3}{*}{2} & \multirow{3}{*}{\begin{tabular}{|l|} 
Tema: \\
Nociones, \\
conceptos y \\
generalizaciones \\
del lenguaje \\
natural al \\
lenguaje \\
algebraico
\end{tabular}} & \multirow{3}{*}{\begin{tabular}{|l|} 
5.Animaplano \\
Aprendizaje \\
basado por \\
juegos
\end{tabular}} & \multirow{3}{*}{$\begin{array}{l}\text { 6.Exposiciones } \\
\text { Aprendizaje por } \\
\text { indagación, } \\
\text { compartido y } \\
\text { cooperativo }\end{array}$} & & Observación \\
\hline & & & & & Rubrica \\
\hline & & & & & Heteroevaluación \\
\hline \multirow[t]{6}{*}{3} & \multirow{6}{*}{$\begin{array}{l}\text { Tema: Noción y } \\
\text { representación } \\
\text { del concepto de } \\
\text { expresiones } \\
\text { algebraicas. } \\
\text { Expresiones } \\
\text { algebraicas a } \\
\text { partir de un } \\
\text { enunciado. }\end{array}$} & & & \multirow{3}{*}{$\begin{array}{l}\text { 7. Juego: Stop } \\
\text { Aprendizaje basado } \\
\text { en juegos }\end{array}$} & Observación \\
\hline & & & & & Rúbrica \\
\hline & & & & & Heteroevaluación \\
\hline & & \multirow{3}{*}{$\begin{array}{l}\text { 8. Tarea: } \\
\text { ejercicios } \\
\begin{array}{l}\text { Aprendizaje por } \\
\text { repetición }\end{array}\end{array}$} & & & $\begin{array}{c}\text { Análisis de } \\
\text { producto }\end{array}$ \\
\hline & & & & & Lista de chequeo \\
\hline & & & & & Heteroevaluación \\
\hline \multirow[t]{3}{*}{4} & \multirow[t]{3}{*}{$\begin{array}{l}\text { Tema: Concepto } \\
\text { y clasificación } \\
\text { de polinomios. }\end{array}$} & & \multirow{3}{*}{$\begin{array}{l}\text { 10. Actividad } \\
\text { kinestésica. } \\
\text { Aprendizaje } \\
\text { basado en APK y } \\
\text { basado en juegos }\end{array}$} & \multirow{3}{*}{$\begin{array}{l}\text { 9. Salir al } \\
\text { tablero } \\
\text { Aprendizaje basado } \\
\text { en ejercicios y } \\
\text { compartido }\end{array}$} & Observación \\
\hline & & & & & Escala valorativa \\
\hline & & & & & Coevaluación \\
\hline \multirow{6}{*}{6} & \multirow{6}{*}{$\begin{array}{l}\text { Tema: Adición } \\
\text { y sustracción de } \\
\text { polinomios } \\
\text { algebraicos }\end{array}$} & \multirow{3}{*}{$\begin{array}{l}\text { 11. Quiz } \\
\text { Aprendizaje por } \\
\text { ejercicios }\end{array}$} & \multirow{3}{*}{$\begin{array}{l}\text { 10. Juego de } \\
\text { tarjetas } \\
\text { Aprendizaje } \\
\text { basado en juegos }\end{array}$} & & $\begin{array}{l}\text { Análisis de } \\
\text { producto }\end{array}$ \\
\hline & & & & & Lista de chequeo \\
\hline & & & & & Heteroevaluación \\
\hline & & \multirow{3}{*}{$\begin{array}{|ll|}\text { 11. Video- } & \\
\text { Quest } & \\
& \\
\text { Aprendizaje } & \\
\text { basado } & \text { en } \\
\text { ejercicios } & \\
\end{array}$} & \multirow{3}{*}{$\begin{array}{l}\text { 12. Tangram } \\
\text { Aprendizaje } \\
\text { basado en juegos }\end{array}$} & & Encuestación \\
\hline & & & & & Cuestionario \\
\hline & & & & & Heteroevaluación \\
\hline
\end{tabular}




\begin{tabular}{|l|l|l|l|l|c|}
\hline 7 & $\begin{array}{l}\text { Tema: } \\
\text { Multiplicación, } \\
\text { potenciación y } \\
\text { de polinomios } \\
\text { algebraicos }\end{array}$ & $\begin{array}{l}\text { 13. Taller } \\
\text { Aprendizaje } \\
\text { basado } \\
\text { ejercicios }\end{array} \quad$ en & $\begin{array}{l}\text { 14.Rompecabez } \\
\text { a (rectángulos) } \\
\text { Aprendizaje } \\
\text { basado en } \\
\text { ejercicios }\end{array}$ & & Encuestación \\
\cline { 3 - 6 }
\end{tabular}

Tabla 2. Matriz de medios evaluativos resumida para la planeación de la enseñanza desde la evaluación.

\subsection{Metodología}

Se realizó una investigación cuantitativa, partiendo de un diseño cuasi-experimental para luego analizar los resultados obtenidos a través de una técnica correlacional de análisis de la información, para esto se decidió usar estadístico T. En los diseños cuasiexperimentales, los sujetos no se asignan al azar a los grupos ni se emparejan, sino que dichos grupos ya están conformados antes del experimento: son grupos intactos (la razón por la que surgen y la manera como se integraron es independiente o aparte del experimento). Por ejemplo, si fueran tres grupos escolares formados con anterioridad a la realización del experimento, y cada uno de ellos constituye un grupo experimental (Sampieri, Fernández, y Baptista, 2014, p.151). La corroboración de una de las hipótesis planteadas en en los objetivos de la investigación, se derivó de los análisis del pre-tests y del pos-tests (misma prueba aplicada al inicio y final del proceso), así como de las encuestas de satisfacción preevento y postevento.

La población estudiantil del grado octavo es de 160 estudiantes y está constituida en tres grupos dentro de la Institución educativa, se decidió trabajar aleatoriamente con dos grupos de los tres, determinando por azar que uno fuera el grupo experimental y el otro el grupo de control. Para desarrollar el proceso se valió de una prueba pretest-postest que se aplicaron a ambos grupos, así como también se aplicó del mismo modo una encuesta preevento y postevento; se aplicaron medios evaluativos formales, semiformales y no formales, así como métodos de enseñanza-aprendizaje que se consideraron apropiados para la intervención con el grupo experimental; con el grupo control el proceso de enseñanzaevaluación fue tradicional implicando un método de enseñanza-aprendizaje expositivo y por repetición, donde únicamente se valió de medios formales tradicionales como: quiz, examen y taller de ejercitación.

El pretest y el postest tuvieron una duración de 50 minutos en cada sesión de su correspondiente aplicación en el proceso; esta prueba tuvo un diseño de pregunta de opción múltiple con única respuesta y se fundamentó en que el estudiante examinará el significado de los conceptos, símbolos y procedimientos de saberes conexos y propios del álgebra, para determinar: falencias, necesidades, fortalezas y alcances. Las encuestas de satisfacción preevento y post-evento, trataron principalmente el grado en que están en acuerdo o desacuerdo los estudiantes con los medios evaluativos con que han sido y no han sido evaluados anteriormente, la frecuencia de su uso en clase, así como también sobre si conocían tipos específicos de medios evaluativos (como los que finalmente se trabajaron en el desarrollo de la investigación); Las encuestas están ligadas a una escala valorativa de 1 a 5 , siendo 1 muy desacuerdo y 5 muy de acuerdo; la realización de la prueba duró 25 minutos en cada sesión de su correspondiente aplicación en el proceso. 
El pretest y las preguntas de clase le sirvió en un inicio al maestro como medio diagnóstico y como técnica para llevar a cabo la planificación de los medios evaluativos. Hay que resaltar que en el desarrollo del proceso, se evidenció que muchos estudiantes poseían debilidades en operaciones básicas como la adición, la multiplicación, la división y la potenciación que fueron reforzadas en la implementación de cada medio evaluativo correspondiente. Se resalta que "cuando los conocimientos, sobre todo los básicos, no están bien comprendidos, seguir con nuevos conocimientos supone un esfuerzo ineficaz que provocará una sensación de fracaso y tensión emocional" (carrillo, 2009, p.4); lo cual implica que los maestros en general, deben aplicar una adecuada prueba diagnóstica, buscando determinar qué temas deben reforzar y socializar para que sus estudiantes se nivelen, y así puedan solucionar las falencias que tienen sobre el tema.

\section{Resultados}

\subsection{Resultados iniciales de la prueba tipo test}

A continuación, se presentan los gráficos estadísticos correspondientes a los resultados del test inicial por preguntas (figura 1 y figura 2) y resultados iniciales; se analizaron las medias para comparar si había diferencias significativas, entre el grupo de control y el experimental, analizando desde el porcentaje correspondiente a la respuesta correcta, a la respuesta incorrecta y a la categoría de no contesta; Se resalta que cada estudiante recibió una nota entre 0 a 5 , obteniendo un valor de 0.41 por cada pregunta correcta.

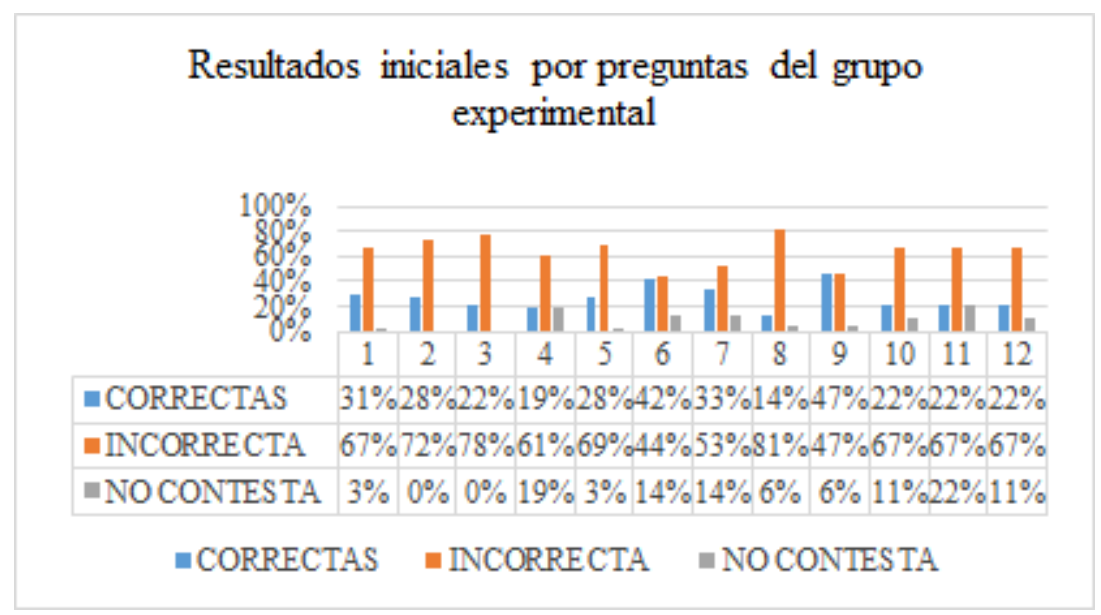

Fig.1. Resultados iniciales por pregunta grupo experimental 


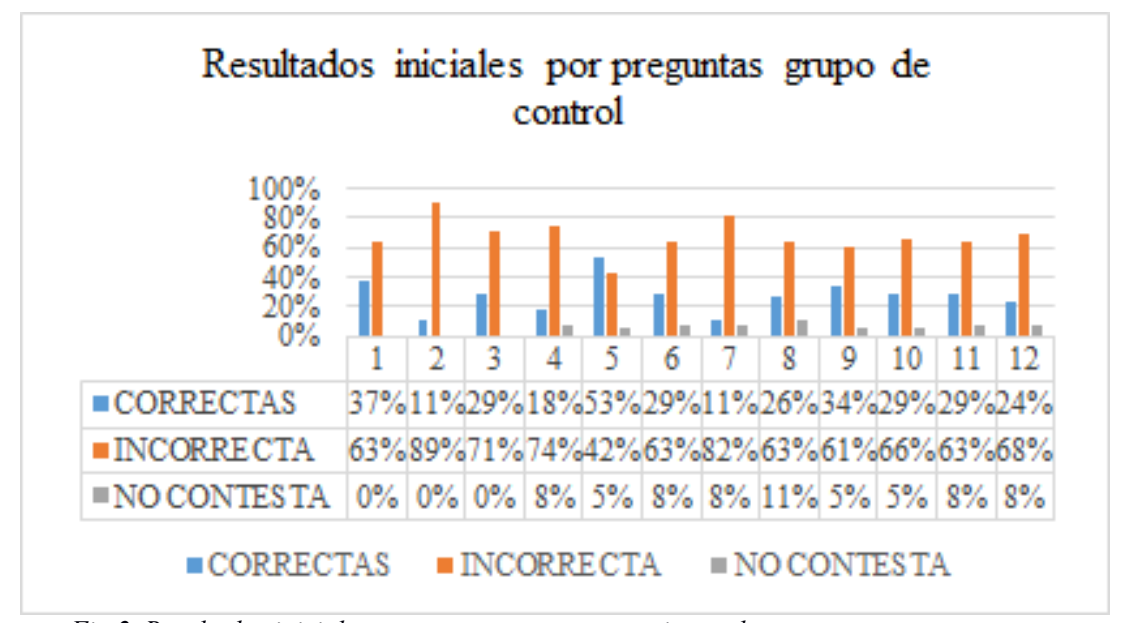

Fig.2. Resultados iniciales por pregunta grupo experimental

Se puede observar en las figuras 1 y 2 que hay ciertas diferencias notables entre los dos grupos; por ejemplo entre las preguntas 5 y 8 mientras el grupo experimental tiene un porcentaje del $28 \%$ y $14 \%$ respectivamente que respondió correcto, mientras que el grupo de control tiene un $53 \%$ y un $26 \%$, con una diferencia entonces del $25 \%$ y $12 \%$; en otras preguntas como la 2, 6, 7 y 9 el grupo experimental los porcentajes en preguntas correctas cuentan con una diferencia de más del $10 \%$ respecto al grupo de control, mientras que en otras preguntas como la 1,3,4,10,11,12 la diferencias entre grupos son menores del 10\%. Sin embargo, es notable que en ambos grupos es mayor el porcentaje de las preguntas incorrectas que las correctas, exceptuando los casos de la pregunta 9 en el grupo de experimental y la 5 en el de control.

\subsection{Análisis con la prueba $t$}

Hipótesis nula: No hay diferencias significativas entre las medias de las notas del grupo experimental y el grupo de control.

Hipótesis alterna: Hay diferencia significativa entre las medias de las notas del grupo experimental y el grupo de control 


\begin{tabular}{|c|c|c|}
\hline \multicolumn{2}{|c|}{ Prueba t para dos muestras suponiendo varianzas iguales } & \multirow[b]{2}{*}{ Grupo experimental } \\
\hline & Grupo de control & \\
\hline Media & 1,381578947 & 1,352777778 \\
\hline Varianza & 0,353435277 & 0,435134921 \\
\hline Observaciones & 38 & 36 \\
\hline Varianza agrupada & 0,393150382 & \\
\hline Diferencia hipotética de las medias & 0 & \\
\hline Grados de libertad & 72 & \\
\hline Estadistico t & 0,197495845 & \\
\hline $\mathrm{P}(\mathrm{T}<=\mathrm{t})$ una cola & 0,421998016 & \\
\hline Valor critico de t (una cola) & 1,666293696 & \\
\hline$P(T<=t)$ dos colas & 0,843996032 & \\
\hline Valor critico de t (dos colas) & 1,993463567 & \\
\hline
\end{tabular}

Fig.3. prueba t para comparar las media de los grupos del pretest

Haciendo el análisis con la prueba t de student, podemos darnos cuenta en los resultados de el nivel de significancia $\mathrm{p}(\mathrm{T} \leq \mathrm{t})$ dos colas es mayor que 0.05 ; por lo tanto podemos afirmar nuestra hipótesis nula, así pues no hay diferencias significativas entre las medias de las notas del grupo experimental y el grupo de control.

\subsection{Resultados del postest}

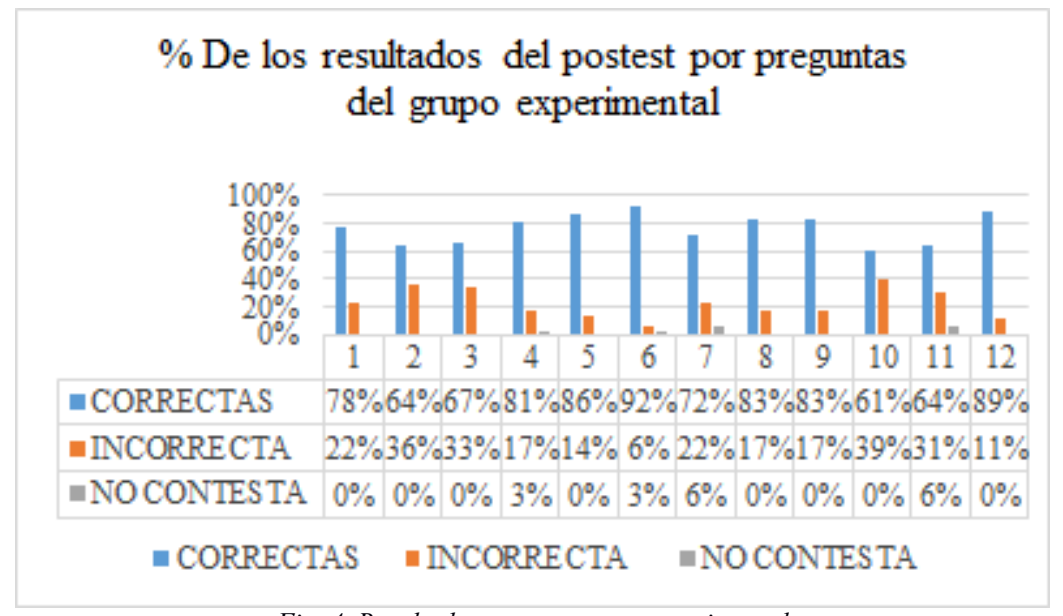

Fig. 4. Resultados postest grupo experimental 


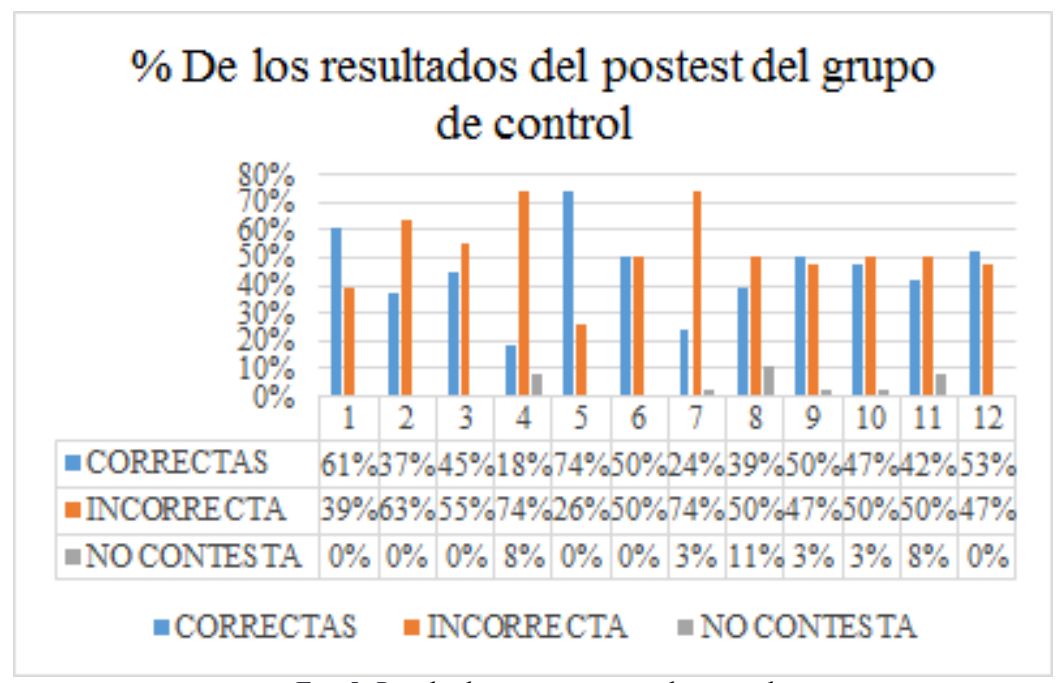

Fig. 5. Resultados postest grupo de control

En las figuras 4 y 5 contienen los resultados del postest, luego de realizar la intervención en el grupo experimental podemos notar que en las preguntas 4,6,7, la diferencia es de más $40 \%$ con respecto al grupo de control; en las demás preguntas la diferencia es de más de un $10 \%$.

\subsection{Resultados después de intervención en el grupo experimental, análisis prueba t}

\begin{tabular}{|l|r|r|}
\hline Prueba t para dos muestras suponiendo varianzas iguales \\
\hline & & \\
\hline & Grupo experimental & Grupo de control \\
\hline Media & 3,827777778 & 2,25 \\
\hline Varianza & 0,525492063 & 0,386351351 \\
\hline Observaciones & 36 & 38 \\
\hline Varianza agrupada & 0,453989198 & \\
\hline Diferencia hipotética de las med & 0 & \\
\hline Grados de libertad & 72 & \\
\hline Estadistico $t$ & 10,06816361 & \\
\hline $\mathrm{P}(\mathrm{T}<=\mathrm{t})$ una cola & $1,10736 \mathrm{E}-15$ & \\
\hline Valor critico de $\mathrm{t}$ (una cola) & 1,666293696 & \\
\hline $\mathrm{P}(\mathrm{T}<=\mathrm{t})$ dos colas & 0,000000 & \\
\hline Valor critico de $\mathrm{t}$ (dos colas) & 1,993463567 & \\
\hline \multicolumn{2}{|l|}{ Fig.6. prueba t para comparar las medias de los grupo en el postest } \\
\hline
\end{tabular}

Hipótesis nula: No hay diferencia significativas entre las medias de las notas del grupo experimental y el grupo de control después de haber aplicado el postest.

Hipótesis alterna: Hay diferencia significativa entre las medias de las notas del grupo experimental y el grupo de control después de haber aplicado el postest. 
Haciendo el análisis con la prueba t de student podemos darnos cuenta en los resultados del nivel de significancia $\mathrm{p}(\mathrm{T}<=\mathrm{t})$ dos colas es menor que 0.05 ; por lo tanto podemos afirmar nuestra hipótesis alterna: hay diferencia significativa entre las medias de las notas del grupo experimental y el grupo de control después de haber aplicado el postest.

A continuación, se presentan los gráficos estadísticos correspondientes a los resultados de la encuesta pre-evento por preguntas (figura 7 y figura 8), y resultados iniciales generales (Figura 9 y figura 10), tanto en el grupo de control como en el experimental. Las figuras identifican la posición de los estudiantes con respecto a la satisfacción que implican los medios evaluativos en su proceso formativo.
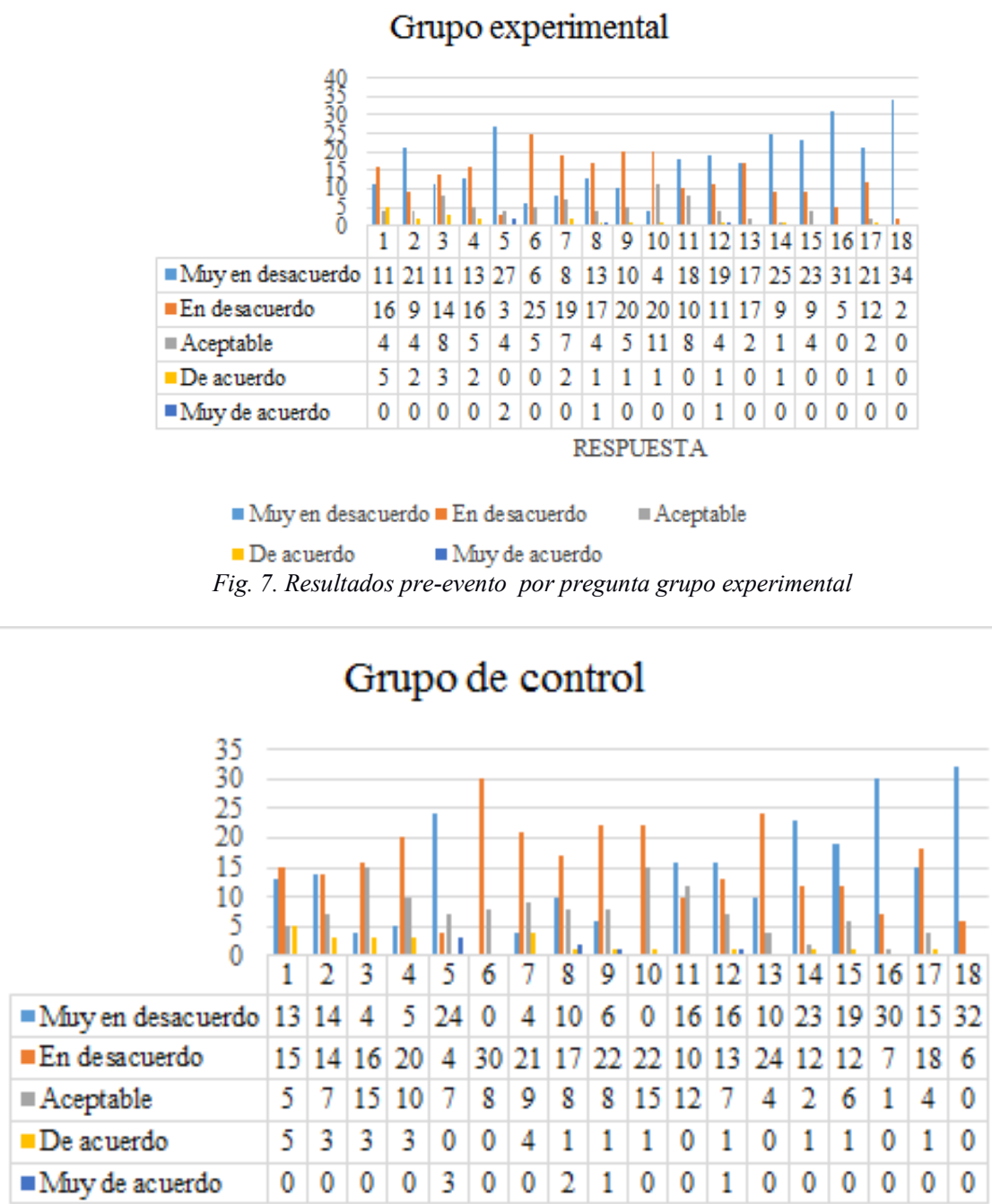

\section{PREGUNTAS}

$\square$ Muy en desacuerdo $\square$ En de sacuerdo $\square$ Aceptable $\square$ De acuerdo $\square$ Muy de acuerdo Fig. 8. Resultados pre-evento Resultados por pregunta grupo de control 
La evaluación formativa en álgebra a través de los medios formales, semiformales y no formales: el caso de expresiones y polinomios algebraicos

En las figuras 7 y 8, se observan ciertas diferencias respecto a cada pregunta, sin embargo se encuentran ciertas similaridades respecto a la encuesta de satisfacción que se les aplica.

\section{Repuestas post-evento grupo experimental}

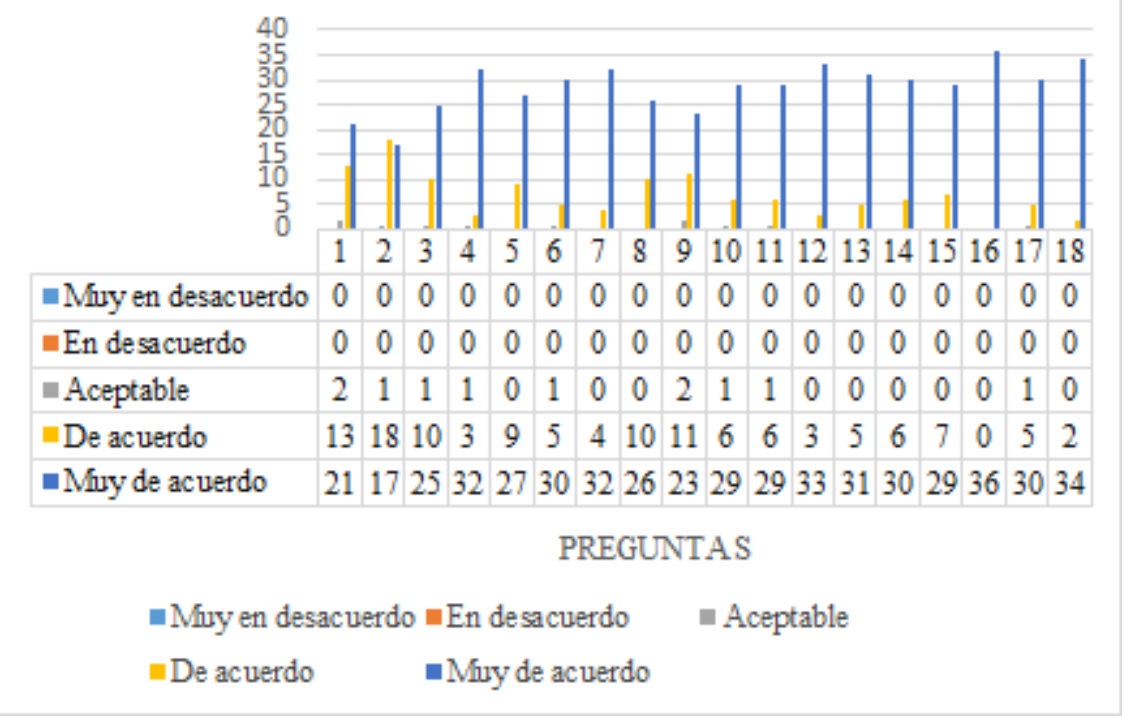

Fig. 9. Resultados post-evento por pregunta del grupo experimental

\section{Resultados del post-eveto del grupo de control}

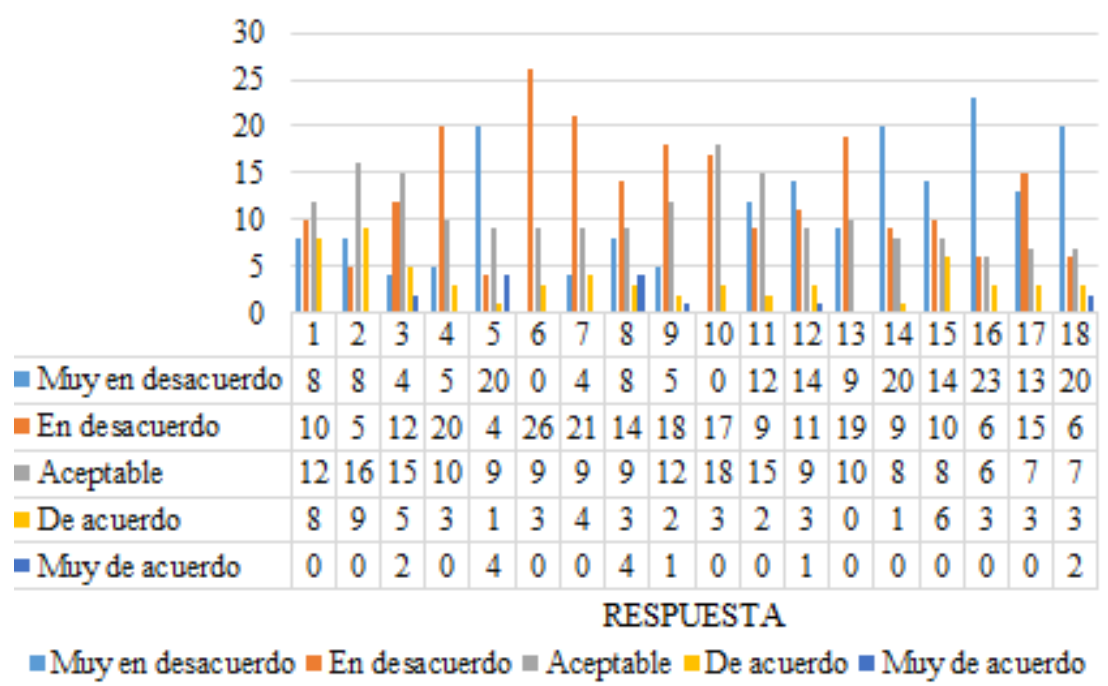

Fig. 10. Resultados post-evento por pregunta del grupo de control 
Los porcentajes evidenciados en las figuras 9, 10 muestran que el grupo experimental está muy de acuerdo con los medios utilizados para evaluar y desarrollar la práctica de enseñanza; los resultados de la encuesta compaginan con los logros adquiridos y las competencias alcanzadas en los postest y el proceso global. La percepción del grupo de control no varió mucho con respecto a su posición inicial en la encuesta de pre-evento.

\section{Conclusiones}

Los resultados obtenidos muestran que esta práctica resultó de forma positiva como estrategia de intervención, por lo que se evidencia que la enseñanza y el desarrollo de un proceso formativo debe plantearse desde la evaluación.

La práctica de evaluación se considera de vital importancia en todo el proceso, tiene un carácter formativo dado que se realizó en aras de la constitución de los logros de aprendizaje y las competencias de la formación para el tema de expresiones y polinomios algebraicos; permitiendo a los profesores valorar, retroalimentar y proalimentar los resultados de las actividades y las consideraciones tenidas en cuenta, más allá de la nota o calificación, permitiéndole a los estudiantes tomar conciencia de la calidad de las propuestas presentadas y de su rol dentro del proceso de evaluación formativa.

\section{Referencias}

Carrillo, B.(2009). “Dificultades en el aprendizaje matemático". Revista Digital, Innovación y experiencia educativas, 1-10. https://archivos.csif.es/archivos/andalucia/ensenanza/revistas/csicsif/revista/pdf/Nu mero_16/BEATRIZ_CARRILLO_2.pdf [Consulta: 10 de marzo 2018]

Colclough, C. (2005). Educación para todos : el imperativo de la calidad. París: Unesco.

Díaz, F y Barriga, A.(2002). Capítulo 8 técnicas e instrumentos de evaluación. http://postgrado.una.edu.ve/evaluacionaprendizajes/paginas/diazbarrigacap8-1.pdf [Consulta: 10 de marzo 2018]

García, A., Aguilera, M., Pérez, M. y Muñoz, G. (2011). “Evaluación de los aprendizajes en el aula, opiniones y prácticas de docentes de primaria en México”. Instituto nacional para la evaluación de la educación. México. https://www.inee.edu.mx/images/stories/2014/Publicaciones_CONPEE/pdf/ev del os_aprend.pdf [Consulta: 12 de marzo 2018]

Hamodi, C., López, V., y López, A. (2015). Medios, técnicas e instrumentos de evaluación formativa y compartida del aprendizaje en educación superior. Perfiles Educativos, vol. XXXVII, 146-161.

Ibarra, M. y Rodríguez, G. (2014). Modalidades participativas de evaluación: Un análisis de la percepción del profesorado y de los estudiantes universitarios.Revista de 
La evaluación formativa en álgebra a través de los medios formales, semiformales y no formales: el caso de expresiones y polinomios algebraicos

Investigación Educativa, 32 (2), 339-361. http://dx.doi.org/10.6018/rie.32.2.172941 [Consulta: 11 de marzo 2018]

Inda, M., Álvarez, S. y Álvarez, R. (2008). “Métodos de evaluación en la enseñanza superior". Revista de Investigación Educativa, 26 (2), 539-552. https://www.redalyc.org/articulo.oa?id=283321909014 [Consulta: 10 de marzo 2018]

Ministerio de Educación Nacional (1998). Serie lineamientos curriculares Matemáticas. Santa Fe de Bogotá, Colombia.

Madaus, G., Airasian, P. (1970). Placement, Formative, Diagnostic, and Summative Evaluation of Classroom Learning. Revista de Investigación Educativa, 25. United States.https://files.eric.ed.gov/fulltext/ED041829.pdf [Consulta: 11 de marzo 2018]

Núñez, P., Bono., R y Suárez, M., "Evaluación formativa en Educación Superior: Impacto en estudiantes con ansiedad a las matemáticas" International Conference on University Teaching and Innovation, CIDUI 2014, 2-4 July 2014, Tarragona, Spain. p 135-141

Pérez, A., Pérez, A. y Hernández H. (2013) "Secuencia didáctica para facilitar la transición entre la aritmética y el álgebra. Universidad Autónoma de Chiapas". En Flores R. (Ed.). Acta Latinoamericana de Matemática Educativa, Vol. 26. México, DF: Colegio Mexicano de Matemática Educativa A. C. y Comité Latinoamericano de Matemática Educativa A. C. (Pérez, A., Pérez, A. y Hernández H. 2013, p. 870) http://funes.uniandes.edu.co/4124/1/PerezSecuenciaALME2013.pdf [Consulta: 11 de marzo 2018]

Rodriguez, G. e Ibarra, M. (2011),e-Evaluación orientada al e-aprendizaje estratégico en educación superior. Madrid, Narcea.

Rodriguez, G. e Ibarra, M. (2012). La evaluación de los procesos y contextos educativos. En Navaridas, F. (Ed.) Procesos y contextos educativos. (p.257-298). Murcia: Genueve Ediciones. España. https://www.researchgate.net/publication/282819028_La_evaluacion_de_los_proc esos y contextos educativos [Consulta: 11 de marzo 2018] ISBN - 978-84940186-8-8

Sampieri, R., Fernández, C., y Baptista, P. (2014). Metodología de la investigación. (6a. ed.). McGraw-Hill Interamericana. México. 